\title{
Phase I Study of Triweekly Nab-Paclitaxel Combined with S-1 in Patients with HER2-negative Metastatic Breast Cancer
}

\author{
KOICHI SAKAGUCHI, KATSUHIKO NAKATSUKASA and TETSUYA TAGUCHI \\ Department of Endocrine and Breast Surgery, \\ Kyoto Prefectural University of Medicine Graduate School of Medical Science, Kyoto, Japan
}

\begin{abstract}
Aim: We conducted a phase I study to determine the maximum tolerated dose (MTD) and recommended dose $(R D)$ of triweekly nanoparticle albumin-bound paclitaxel (nab-paclitaxel) and $S-1$ combination therapy in patients with human epidermal growth factor receptor 2 (HER2)negative metastatic breast cancer (MBC). Patients and Methods: This study was carried out with a 3+3 dose escalation design; patients with HER2-negative MBC received nab-paclitaxel at $180-260 \mathrm{mg} / \mathrm{m}^{2}$ on day 1 and $S-1$ at $65-80 \mathrm{mg} / \mathrm{m}^{2}$ daily on days $1-14$, repeated every 3 weeks. Results: The MTD was level $3\left(260 \mathrm{mg} / \mathrm{m}^{2}\right.$ nab-paclitaxel with $\left.80 \mathrm{mg} / \mathrm{m}^{2} \mathrm{~S}-1\right)$ and the $R D$ was level $2\left(260 \mathrm{mg} / \mathrm{m}^{2} \mathrm{nab}\right.$ paclitaxel with $\left.65 \mathrm{mg} / \mathrm{m}^{2} \mathrm{~S}-1\right)$. Dose-limiting toxicity was observed in two patients at level 3, who had grade 4 neutropenia and grade 3 myalgia, respectively. Conclusion: The response rate was $66.7 \%$. The clinical benefit rate was $77.8 \%$. Our study shows the efficacy and the feasibility of this combination therapy.
\end{abstract}

Treatment for metastatic breast cancer (MBC) is rarely curative, therefore palliating symptoms, prolonging survival, and maintaining/improving quality of life are usually the main treatment goals. Although several alternatives are available, currently no gold standard exists for chemotherapy regimens for this population (1). Thus, new therapeutic agents, especially against refractory metastatic tumors, are needed.

Combination chemotherapy regimens offer a statistically significant advantage for survival, tumor response and time to progression in women with $\mathrm{MBC}$, but they also have more toxicity. In a phase I study, the combination of carboplatin

Correspondence to: Koichi Sakaguchi, Department of Endocrine and Breast Surgery, Kyoto Prefectural University of Medicine Graduate School of Medical Science, 465 Kajii-cho, KawaramachiHirokoji, Kamigyo ku, Kyoto 602-8566, Japan. Tel: +81 752515534, Fax: +81 752510270, e-mail: ksak@koto.kpu-m.ac.jp

Key Words: Breast cancer, Nab-paclitaxel, S-1, metastatic, HER2negative, phase I study. and nanoparticle albumin-bound paclitaxel (nab-paclitaxel) was found to be well tolerated and active in heavily pretreated patients with solid tumors, including breast cancer (2). An unresolved question is whether combination regimens are more effective than single agents given sequentially (3); we believe that combination chemotherapy is indicated for patients with visceral crises (the presence of lymphangitic lung metastases, bone marrow involvement, carcinomatous meningitis, or significant liver metastases). In a phase III clinical trial that involved MBC, O'Shaughnessy et al. demonstrated that capecitabine/docetaxel combination therapy was more effective than single-agent docetaxel in terms of overall survival (OS) (4).

Nab-paclitaxel is a novel Cremophor-free nanometer-sized albumin-bound paclitaxel particle initially developed to avoid the allergy associated with use of polyethylated castor oil. Administered as a colloidal suspension of 130-nanometer particles, nab-paclitaxel allows a safe infusion of significantly higher doses of paclitaxel with shorter infusion schedules, without premedication.

$\mathrm{S}-1$, an oral fluoropyrimidine anticancer agent, contains tegafur, a pro-drug of 5-fluorouracil (5-FU), as a base component, as well as two modulators of 5-FU: gimeracil and potassium oxonate. Gimeracil inhibits dihydro-pyrimidine dehydrogenase, an enzyme that degrades 5-FU, thus maintaining high blood levels of 5-FU. Potassium oxonate is a selective inhibitor of orotate phosphoribosyltransferase and reduces gastrointestinal toxicity through the suppression of 5FU activation and 5-fluoro-2'-deoxyuridine-5'-monophosphate synthesis in gastrointestinal mucosa.

Nukatsuka et al. showed that S-1 and paclitaxel had a synergistic effect on tumor regression in a mouse model of human breast cancer (5). In phase III clinical trials of MBC, Gradishar et al. reported that triweekly nab-paclitaxel had greater efficacy and a more favorable safety profile than with triweekly paclitaxel (6). From the basic study results and clinical information, we anticipated that S-1 and nab-paclitaxel would synergistically yield greater efficacy, hence we conducted a phase I dose-escalation study using a combination of nab-paclitaxel and S-1 to evaluate its efficacy and safety. 
Table I. Nab-paclitaxel and S-1 combination regimens. Nab-paclitaxel was given on day 1 and $S-1$ daily on days 1-14. The regimen was repeated every 3 weeks.

\begin{tabular}{lcc}
\hline Level & Nab-paclitaxel & $\mathrm{S}-1$ \\
\hline 0 & $180 \mathrm{mg} / \mathrm{m}^{2}$ & $65 \mathrm{mg} / \mathrm{m}^{2}$ \\
1 & $220 \mathrm{mg} / \mathrm{m}^{2}$ & $65 \mathrm{mg} / \mathrm{m}^{2}$ \\
2 & $260 \mathrm{mg} / \mathrm{m}^{2}$ & $65 \mathrm{mg} / \mathrm{m}^{2}$ \\
3 & $260 \mathrm{mg} / \mathrm{m}^{2}$ & $80 \mathrm{mg} / \mathrm{m}^{2}$ \\
\hline
\end{tabular}

\section{Patients and Methods}

Patient eligibility. Patients were eligible for this study if they had histologically confirmed breast cancer; were aged 20-64 years; had an Eastern Cooperative Oncology Group performance status of 0 or 1 ; and had a survival expectancy of at least 6 months. Other eligibility criteria were: no severe dysfunction of major organs such as the bone marrow, heart, lung, liver, or kidney; leukocyte count of $\geq 4000 / \mathrm{mm}^{3}$; neutrophil count of $\geq 2000 / \mathrm{mm}^{3}$; hemoglobin concentration of $\geq 9.0 \mathrm{~g} / \mathrm{dl}$; platelet count of $\geq 10.0 \times 10^{4} / \mathrm{mm}^{3}$; serum total bilirubin concentration of $\leq 1.5 \mathrm{mg} / \mathrm{dl}$; serum aspartate aminotransferase concentration of $<100 \mathrm{IU} / \mathrm{l}$; serum alanine aminotransferase of $<100 \mathrm{IU} / \mathrm{l}$; serum creatinine clearance of $\geq 60$ $\mathrm{ml} / \mathrm{min}$ as determined from 24 -h urine collection, or predicted creatinine clearance calculated using the Cockcroft-Gault formula. All patients gave their written informed consent to participate in the study. Patients with a history of taxane or S-1 chemotherapy for MBC and those who had experienced grade 2 or more peripheral neuropathy before enrolment were excluded. All aspects of the study were approved by the Ethics Committee of Kyoto Prefectural University of Medicine on February 29, 2012 (RBMR-C-1077-2). This study was registered with the UMIN Clinical Trial Registry (UMIN-CTR, UMIN 000016377).

Treatment regimen and dose-escalation schedule. Dosage schedules at each dose level are shown in Table I. This study was carried out with a $3+3$ design in a sequential order of level 1,2, 3. If none of the three patients experienced a dose-limiting toxicity (DLT) at a particular level, the dose was escalated to the next level in subsequent patients. If two or three out of the three patients experienced a DLT, the level was not escalated further and the level was identified as the MTD. If only one out of the three patients experienced a DLT, three additional patients were to be recruited for the same level; so if only one patient out of the six experienced a DLT, the dose was escalated to the next level, but if two or more patients experienced a DLT, the level was not escalated further and the level was identified as the MTD. The dose level immediately below the MTD was defined as the recommended dose (RD). If no patients experienced a DLT even at maximal dose level 3, then dose level 3 was identified as the RD.

DLT was defined as the occurrence of any of the following during cycle 1: grade 4 decrease in platelet count; grade 3 decrease in platelet count that required blood transfusion; febrile neutropenia with a neutrophil count of $<500 / \mathrm{mm}^{3}$ and pyrexia of $\geq 38.5^{\circ} \mathrm{C}$; decrease in grade 4 neutrophil count for $\geq 7$ days; or grade $\geq 3$ nonhematological toxicities.
Table II. Patient characteristics.

\begin{tabular}{ll}
\hline Characteristic & No. of patients $(\%)$ \\
\hline Median age (range), years & 56 (46-65) \\
ECOG PS & 9 \\
0 & 0 \\
1 & \\
Hormonal status & 6 \\
ER-positive and/or PgR-positive & 3 \\
ER-negative and PgR-negative & \\
Metastatic site & 2 \\
Lung & 7 \\
Bone & 2 \\
Liver & 6 \\
Distant lymph nodes & 1 \\
Other & \\
Prior chemotherapy for MBC & 5 \\
0 & 2 \\
2 & 1 \\
3 & 1 \\
6 & \\
\hline
\end{tabular}

ECOG: Eastern Cooperative Oncology Group; ER: estrogen receptor; PgR: progesterone receptor; PS: performance status; MBC: metastatic breast cancer.

Response evaluation and toxicity. The safety and tolerability of the S-1 and nab-paclitaxel combination therapy were assessed according to the National Cancer Institute Common Toxicity Criteria (CTC) (version 4.0) (7). Antitumor response evaluations were carried out every two cycles. Objective tumor response was evaluated according to the Response Evaluation Criteria in Solid Tumors (RECIST) version $1.1(8)$.

Study objectives. Our primary end points were to estimate the MTD and RD of the combination therapy of triweekly nab-paclitaxel and S-1 in patients with MBC. The secondary end points were to evaluate the time to treatment failure (TTF), OS, and safety.

\section{Results}

Patient characteristics. During 2012 to 2015, nine patients were enrolled in this phase I trial. Their characteristics are summarized in Table II. The median age was 56 years (range: 46-65 years). Five patients had stage 4 disease. Four patients had previously received 2-6 chemotherapies, including anthracycline and taxane.

Dose escalation and determination of MTD and RD. The dose-escalation scheme, including the number of cycles, patients, and DLTs by dose level, is summarized in Table III. As no DLTs were observed at dose levels 1 and 2, doses of nab-paclitaxel and S-1 were escalated to level 3. No DLTs were observed in the first patient who received dose level 3; however, the next two patients experienced DLTs, grade 4 neutropenia persisting for 7 days, and grade 3 myalgia, 
Table III. Dose-escalation scheme.

\begin{tabular}{|c|c|c|c|c|c|c|c|c|c|}
\hline \multirow[b]{2}{*}{ Level } & \multirow[b]{2}{*}{$\begin{array}{l}\text { No. of } \\
\text { patients }\end{array}$} & \multirow[b]{2}{*}{$\begin{array}{c}\text { Nab-paclitaxel } \\
\text { dose }\left(\mathrm{mg} / \mathrm{m}^{2}\right)\end{array}$} & \multirow[b]{2}{*}{$\mathrm{mg} / \mathrm{m}^{2}$} & \multicolumn{2}{|c|}{ S-1 } & \multicolumn{4}{|c|}{ No. of cycles } \\
\hline & & & & $\mathrm{BSA}<1.25 \mathrm{~m}^{2}$ & $\begin{array}{c}1.25 \mathrm{~m}^{2} \\
\text { BSA }<1.5 \mathrm{~m}^{2}\end{array}$ & $\mathrm{BSA} \geq 1.5 \mathrm{~m}^{2}$ & Median & Range & $\begin{array}{l}\text { No. of patients } \\
\text { with DLT }\end{array}$ \\
\hline 0 & 0 & 180 & 65 & 100 & 160 & 200 & & & \\
\hline 1 & 3 & 220 & 65 & 100 & 160 & 200 & 4 & $4-9$ & 0 \\
\hline 2 & 3 & 260 & 65 & 100 & 160 & 200 & 13 & $7-26$ & 0 \\
\hline 3 & 3 & 260 & 80 & 160 & 200 & 240 & 3 & $1-13$ & 2 \\
\hline
\end{tabular}

BSA: Body surface area ; DLT: dose-limiting toxicity.

Table IV. Efficacy of combination therapy by response evaluation criteria in solid tumors(8) guidelines.

\begin{tabular}{lcccc}
\hline Response & Level 1 $(\mathrm{n}=3)$ & Level 2 $(\mathrm{n}=3)$ & Level 3 $(\mathrm{n}=3)$ & Total $(\mathrm{n}=9)$ \\
\hline CR & 0 & 0 & 0 & 0 \\
PR & 2 & 2 & 2 & 6 \\
SD & 0 & 1 & 0 & 1 \\
PD & 1 & 0 & 0 & 1 \\
NE & 0 & 0 & 1 & 1 \\
Response rate (CR+PR) & $66.7 \%$ & $66.7 \%$ & $66.7 \%$ & $66.7 \%(95 \% \mathrm{CI}=29.9-92.5 \%)$ \\
Clinical benefit rate (CR+PR+SD>16 weeks) & $66.7 \%$ & $100.0 \%$ & $66.7 \%$ & $77.8 \%(95 \% \mathrm{CI}=45.0-95.9 \%)$ \\
\hline
\end{tabular}

CR, Complete response; NE, not evaluable; PD, progressive disease; PR, partial response; SD, stable disease; CI: confidence interval.

respectively, at cycle 1 of level 3 . Therefore, the MTD was defined as level 3 and the RD was defined as level 2.

Toxicities. Nine patients received a total of 84 cycles of combination chemotherapy. The median number of cycles administered per patient was 7 (range $=1-26$ ). The overall relative dose intensities were $71.2 \%$ for nab-paclitaxel and $79.2 \%$ for S1. The major reasons for dose reductions were peripheral neuropathy for nab-paclitaxel $(n=6 ; 66.7 \%)$, and fatigue for $\mathrm{S}-1$ $(\mathrm{n}=2 ; 22.2 \%)$. Among hematological toxicities, neutropenia was only observed in three patients $(33.3 \%)$. Grade 4 neutropenia was observed in one patient after day 8 of the first cycle, and was a DLT. The most common grade 1 or 2 non-hematological toxicities were alopecia in all nine patients (100\%), peripheral neuropathy in six $(66.7 \%)$, watery eyes in three $(33.3 \%)$, and fatigue in three $(33.3 \%)$. Grade 3 non-hematological toxicities included peripheral neuropathy in two patients $(22.2 \%)$, myalgia in one $(11.1 \%$, after day 8 of the first cycle, which was defined as a DLT), and watery eyes in one $(11.1 \%)$ who had lacrimal tube placement surgery after three cycles.

The most common reasons for discontinuing therapy were disease progression $(n=4,44.4 \%)$, peripheral neuropathy $(n=2,22.2 \%)$, and watery eyes $(n=1,11.1 \%)$.

Efficacy. Changes in tumor size from baseline to maximal tumor shrinkage are shown in Figure 1. Response rate (RR)

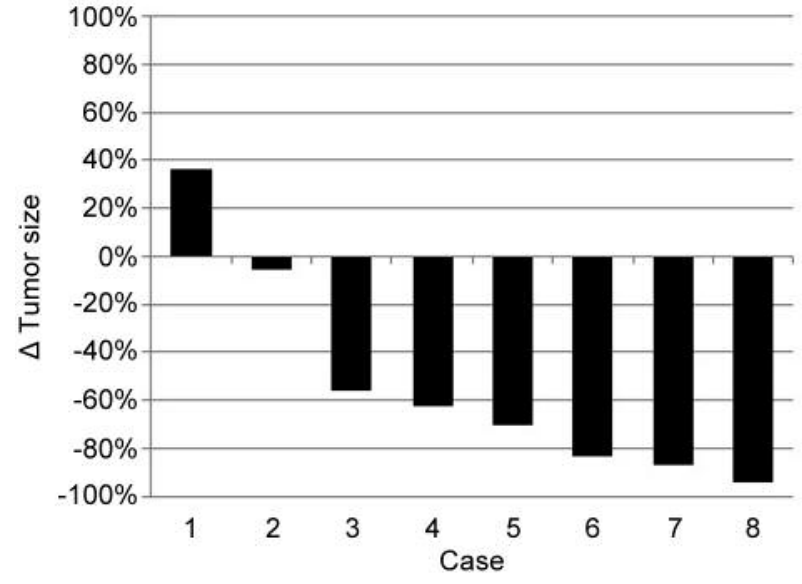

Figure 1. Waterfall plot of changes (by percentage) in tumor size from baseline to maximal tumor shrinkage with regard to overall response. Case 9 had an immeasurable lesion.

and clinical benefit rate [complete response $(\mathrm{CR})+$ partial response $(\mathrm{PR})+$ stable disease $(\mathrm{SD})$ for $\geq 16$ weeks] are shown in Table IV. Eight out of the nine patients had measurable lesions (as defined by RECIST version 1.1). Responses in the nine patients included no CR, six PRs, one $\mathrm{SD}$, one progressive disease (PD), and one patient who was 
not evaluable, for a RR of $66.7 \%$ (95\% confidence interval $=29.9-92.5 \%$ ). The clinical benefit rate was $77.8 \%$ (95\% confidence interval $=45.0-95.9 \%)$.

\section{Discussion}

The median survival from diagnosis of MBC is 2-3 years, with $5-10 \%$ surviving beyond 5 years. The goal of treatment should be to prolong survival while controlling symptoms and minimizing toxicity. Combined chemotherapy regimens are commonly thought to be more active, with improved tumor response and progression rates and thus likely to offer longer OS. Recently, two large individual trials also showed survival benefits for combination regimens compared with single agents. In the first trial, docetaxel plus capecitabine led to a better objective response rate (42\% vs. $30 \%$, $p=0.006)$, median OS (14.5 vs. 11.5 months, $p=0.0126)$ and no measurable decline in quality of life (9). In the second trial, a randomized phase III study compared OS and safety of gemcitabine plus paclitaxel versus paclitaxel monotherapy in patients with advanced breast cancer after failure of prior anthracycline-containing adjuvant chemotherapy. Approval was based on a phase III trial of 529 patients, in whom this combination produced a better RR (41.4\% vs. $26.2 \%)$, and longer median OS (18.6 months $v s .15 .8$ months, $p=0.049$ ) than paclitaxel alone (10). These trials indicate that higher response rates bring about longer survival. As we found a high response rate with nab-paclitaxel plus S-1 therapy, this regimen is promising. Whether nab-paclitaxel plus S-1 therapy can prolong OS is unclear, but should be addressed in a phase III trial comparing nab-paclitaxel plus S-1 with nab-paclitaxel or S-1 alone.

Among non-hematological toxicities, we focused on peripheral neuropathy and watery eyes. Nab-paclitaxel often has non-hematological adverse events, including peripheral neuropathy and muscle pain $(11,12)$. As peripheral neuropathy can lead to dose reduction or complete discontinuation of a treatment, methods of controlling these effects in order to maintain recommended dose levels are critical. Treatments such as the 'frozen glove' to reduce the drug's ability to reach the skin are being tried, (13), but no generally accepted method to address peripheral neuropathy is currently available (14).

Watery eyes area significant clinical problem that reduces the patient's quality of life, and are a common nonhematological adverse event associated with S-1 therapy (15, 16). In our study, watery eyes was observed in four patients. Docetaxel is the drug best-known to cause watery eyes (1722). A recent study indicated that capecitabine maintenance therapy is a potential treatment strategy for patients with MBC patients (23). As S-1 and capecitabine are the same type of oral chemotherapy drug, $\mathrm{S}-1$ treatment is a plausible maintenance therapy for MBC patients who respond to nabpaclitaxel combined with S-1 chemotherapy.
In conclusion, this clinical trial of triweekly nab-paclitaxel with S-1 showed that the MTD of the combination therapy was reached at level 3, and RD was set as $260 \mathrm{mg} / \mathrm{m} 2$ nabpaclitaxel intravenously injected on day 1 of a 21-day cycle, in combination with $65 \mathrm{mg} / \mathrm{m} 2$ oral S-1 for 14 days, followed by 1 week of rest. Further investigation of the long-term safety and efficacy in phase II and ensuing trials is needed.

\section{Conflicts of Interest}

The Authors declare no conflicts of interest in regard to this study.

\section{Acknowledgements}

The Authors gratefully acknowledge the participating patients and their families for their invaluable contribution. They also thank Edanz (Fukuoka, Japan) for English language editing.

\section{References}

1 Briest S and Stearns V: Chemotherapeutic strategies for advanced breast cancer. Oncology 21: 1325-1335; 1340, 2007.

2 Ando M, Yonemori K, Katsumata N, Shimizu C, Hirata T, Yamamoto H, Hashimoto K, Yunokawa M, Tamura K and Fujiwara Y: Phase I and pharmacokinetic study of nab-paclitaxel, nanoparticle albumin-bound paclitaxel, administered weekly to Japanese patients with solid tumors and metastatic breast cancer. Cancer Chemother Pharmacol 69: 457-465, 2012.

3 Cardoso F, Bedard PL, Winer EP, Pagani O, Senkus-Konefka E, Fallowfield LJ, Kyriakides S, Costa A, Cufer T, Albain KS and Force E-MT: International guidelines for management of metastatic breast cancer: combination $v s$. sequential single-agent chemotherapy. J Natl Cancer Inst 101: 1174-1181, 2009.

4 O'Shaughnessy J, Miles D, Vukelja S, Moiseyenko V, Ayoub JP, Cervantes G, Fumoleau P, Jones S, Lui WY, Mauriac L, Twelves C, Van Hazel G, Verma S and Leonard R: Superior survival with capecitabine plus docetaxel combination therapy in anthracycline-pretreated patients with advanced breast cancer: phase III trial results. J Clin Oncol 20: 2812-2823, 2002.

5 Nukatsuka M, Fujioka A, Nakagawa F, Oshimo H, Kitazato K, Uchida J, Sugimoto Y, Nagayama $\mathrm{S}$ and Fukushima M: Antimetastatic and anticancer activity of S-1, a new oral dihydropyrimidine-dehydrogenase-inhibiting fluoropyrimidine, alone and in combination with paclitaxel in an orthotopically implanted human breast cancer model. Int J Oncol 25: 15311536, 2004.

6 Gradishar WJ, Tjulandin S, Davidson N, Shaw H, Desai N, Bhar P, Hawkins $M$ and O'Shaughnessy J: Phase III trial of nanoparticle albumin-bound paclitaxel compared with polyethylated castor oil-based paclitaxel in women with breast cancer. J Clin Oncol 23: 7794-7803, 2005.

7 National Institute of Cancer: Common Terminology Criteria for Adverse Events ( CTCAE ). NIH Publ 2009: 0-71, 2010.

8 Eisenhauer EA, Therasse P, Bogaerts J, Schwartz LH, Sargent D, Ford R, Dancey J, Arbuck S, Gwyther S, Mooney M, Rubinstein L, Shankar L, Dodd L, Kaplan R, Lacombe D and Verweij J: New response evaluation criteria in solid tumours: Revised RECIST guideline (version 1.1). Eur J Cancer 45: 228247, 2009. 
9 Verma S, Maraninchi D, O'Shaughnessy J, Jamieson C, Jones S, Martin M, McKendrick J, Miles D, Twelves C and Hornberger $\mathrm{J}$ : Capecitabine plus docetaxel combination therapy. Cancer 103: 2455-2465, 2005.

10 Albain KS, Nag SM, Calderillo-Ruiz G, Jordaan JP, Llombart AC, Pluzanska A, Rolski J, Melemed AS, Reyes-Vidal JM, Sekhon JS, Simms L and O'Shaughnessy J: Gemcitabine plus Paclitaxel versus Paclitaxel monotherapy in patients with metastatic breast cancer and prior anthracycline treatment. J Clin Oncol 26: 3950-3957, 2008.

11 McCarthy AL, Shaban RZ, Gillespie K and Vick J: Cryotherapy for docetaxel-induced hand and nail toxicity: randomised control trial. Support Care Cancer 22: 1375-1383, 2014.

12 Yamada K, Yamamoto N, Yamada Y, Mukohara T, Minami H and Tamura T: Phase I and pharmacokinetic study of ABI-007, albumin-bound paclitaxel, administered every 3 weeks in Japanese patients with solid tumors. Jpn J Clin Oncol 40: 404$411,2010$.

13 Wolf S, Barton D, Kottschade L, Grothey A and Loprinzi C: Chemotherapy-induced peripheral neuropathy: prevention and treatment strategies. Eur J Cancer 44: 1507-1515, 2008.

14 Leandro-Garcia LJ, Inglada-Perez L, Pita G, Hjerpe E, Leskela S, Jara C, Mielgo X, Gonzalez-Neira A, Robledo M, AvallLundqvist E, Green H and Rodriguez-Antona C: Genome-wide association study identifies ephrin type A receptors implicated in paclitaxel induced peripheral sensory neuropathy. J Med Genet 50: 599-605, 2013.

15 Kanazawa T, Tanakaya $\mathrm{K}$ and Takeuchi H: Two cases of lacrimal duct occlusion caused by S-1 therapy in patients with gastrointestinal cancer. J Jpn Coll Surg 33: 150-154, 2008.

16 Prasad S, Kamath GG and Phillips RP: Lacrimal canalicular stenosis associated with systemic 5-fluorouacil therapy. Acta Ophthalmol Scand 78: 110-113, 2000.

17 Esmaeli B, Ahmadi MA, Rivera E, Valero V, Hutto T, Jackson DM and Newman RA: Docetaxel secretion in tears: association with lacrimal drainage obstruction. Arch Ophthalmol 120: 1180$1182,2002$.
18 Esmaeli B, Hortobagyi GN, Esteva FJ, Booser D, Ahmadi MA, Rivera E, Arbuckle R, Delpassand E, Guerra L and Valero V: Canalicular stenosis secondary to weekly versus every-3-weeks docetaxel in patients with metastatic breast cancer. Ophthalmology 109: 1188-1191, 2002.

19 Esmaeli B, Amin S, Valero V, Adinin R, Arbuckle R, Banay R, Do KA and Rivera E: Prospective study of incidence and severity of epiphora and canalicular stenosis in patients with metastatic breast cancer receiving docetaxel. J Clin Oncol 24: 3619-3622, 2006.

20 Esmaeli B, Valero V, Ahmadi MA and Booser D: Canalicular stenosis secondary to docetaxel (taxotere): a newly recognized side effect. Ophthalmology 108: 994-995, 2001.

21 Kintzel PE, Michaud LB and Lange MK: Docetaxel-associated epiphora. Pharmacotherapy 26: 853-867, 2006.

22 Leyssens B, Wildiers H, Lobelle JP, Gillis A, Paridaens R and Mombaerts I: A double-blind randomized phase II study on the efficacy of topical eye treatment in the prevention of docetaxelinduced dacryostenosis. Ann Oncol 21: 419-423, 2010.

23 Gligorov J, Doval D, Bines J, Alba E, Cortes P, Pierga JY, Gupta V, Costa R, Srock S, de Ducla S, Freudensprung U and Mustacchi G: Maintenance capecitabine and bevacizumab versus bevacizumab alone after initial first-line bevacizumab and docetaxel for patients with HER2-negative metastatic breast cancer (IMELDA): a randomised, open-label, phase 3 trial. Lancet Oncol 15: 1351-1360 2014.
Received October 13, 2016

Revised November 3, 2016

Accepted November 8, 2016 